\title{
A New Delay-Dependent Stability Condition for Stochastic Neural Networks of Neutral-Type with Multiple Discrete and Unbounded Distributed Delays
}

\author{
Guo-Quan Liu ${ }^{* 1,2}$, Simon X.Yang ${ }^{1,3}$, Yi Chai ${ }^{1}$ and Wei Fu ${ }^{1}$ \\ ${ }^{1}$ College of Automation, Chongqing University, No.174, Shazhengjie, Shapingba, Chongqing, 400044 Chongqing, China \\ ${ }^{2}$ School of Mechanical and Electronic Engineering, East China Institute of Technology, 330013 Nanchang, Jiangxi, China \\ ${ }^{3}$ School of Engineering, University of Guelph, Guelph, Ontario, Canada
}

Received: 12 Oct. 2012, Revised: 5 Jan. 2013, Accepted: 7 Jan. 2013

Published online: 1 Jun. 2013

\begin{abstract}
This paper deals with the delay-dependent asymptotic stability analysis problem for stochastic neural networks of neutraltype with mixed delays. The mixed delays comprise both multiple discrete and unbounded distributed delays. To the best of the authors' knowledge, till now, the asymptotic stability problem for this class of neural networks has not yet been solved since neutral-type delays are considered in this paper. The main objective of this paper is to fill this gap. By using Lyapunov-Krasovskii functional method and the linear matrix inequality (LMI) technique, a novel sufficient condition is derived to guarantee the global asymptotic stability of the equilibrium point in the mean square. In particular, the proposed stability condition is presented in terms of LMI, which can be easily solved by some standard numerical packages. In addition, an example is given to show the effectiveness of the obtained result.
\end{abstract}

Keywords: Delay-dependent stability, stochastic neural networks, linear matrix inequality, neutral-type, unbounded distributed delays, multiple discrete delays

\section{Introduction}

As is well-known, artificial neural networks are computational modeling tools that have found extensive acceptance in many disciplines for modeling complex real-world problems [1]. In addition, owing to the fact that in biological and artificial neural networks, there inevitably exist integration and communication delays which may lead to bifurcation, oscillation, divergence, instability, or other poor performances $[2,3,4]$. Therefore, the study of neural networks with time delays has become a subject of intensive research activity, and the corresponding research can be seen in $[3,4,5,6,7,8,9,10$, $11,12,13,14,15,16]$. However, in these publications, most research on delayed neural networks has been restricted to simple cases of discrete delays. Since a neural network usually has a spatial nature due to the presence of an amount of parallel pathways of a variety of axon sizes and lengths, it is desired to model them by introducing distributed delays. Therefore, both discrete and distributed delays should be taken into account when modeling a realistic neural network $[10,11,12]$. In particular, the stability problems of neural networks with multiple discrete delays have received much attention in the last few years, see $[13,14,15]$ and their references.

In a real neural networks system, stochastic disturbances are nearly inevitable owing to various reasons such as random fluctuations in the release of neurotransmitters or thermal noise in electronic devices $[16,17]$. Therefore, it is significant to consider stochastic effects to the stability property of delayed neural networks. In recent years, considerable efforts have been devoted to the asymptotic stability analysis of stochastic neural networks with time delays $[18,19,20]$. For example, in [20], the authors investigated the global asymptotic stability of stochastic recurrent neural networks with multiple discrete and unbounded distributed delays, and a new sufficient condition was presented by employing a Lyapunov-Krasovskii functional combined with the LMI approach. It is noticed that neutral-type delays are not considered in their study. However, neutral-type delays occurring in the interaction

\footnotetext{
*Corresponding author e-mail: guoquanliu1982@hotmail.com
} 
between neurons will affect the stability of networks by creating oscillation or unstable phenomena. So, the stability of with neutral-type delays should be a focused topic of theoretical as well as practical importance. Up to now, the asymptotic stability analysis for stochastic neural networks of neutral-type with multiple discrete and unbounded distributed delays has not been investigated, which motives our research.

This paper is concerned with the problem of global asymptotic stability stability for stochastic neural networks of neutral-type with multiple discrete and unbounded distributed delays. Based on the Lyapunov stability theory and the LMI technique, a new delay-dependent stability condition is obtained in terms of LMI. The proposed model of neural networks is quite general since many factors such as stochastic perturbations, multiple discrete and unbounded distributed delays are considered in this paper. Finally, an example is provided to demonstrate the effectiveness of the proposed stability criteria.

\section{Problem Statements}

In this section, we consider the following stochastic neural networks of neutral-type with multiple discrete and distributed time-varying delays.

$$
\begin{aligned}
& d[y(t)-D y(t-h(t))]=[-C y(t)+B(g(y(t)) \\
& +\sum_{k=1}^{r} W^{(k)} g\left(y\left(t-\tau_{k}(t)\right)\right) \\
& \left.+A \int_{-\infty}^{t} k(t-s) g(y(s)) d s\right] d t \\
& +\sigma\left(t, g(y(t)), g\left(y\left(t-\tau_{1}(t)\right)\right)\right. \\
& \left., g\left(y\left(t-\tau_{2}(t)\right)\right), \ldots, g\left(y\left(t-\tau_{r}(t)\right)\right)\right) d \omega(t)
\end{aligned}
$$

where $y(t)=\left[y_{1}(t), y_{2}(t), \ldots, y_{n}(t)\right]^{T} \in \Re^{n}$ is the neuron state vector, $C=\operatorname{diag}\left\{c_{1}, c_{2}, \ldots, c_{n}\right\}$ is a positive diagonal matrix. $B \in \mathfrak{R}^{n \times n}, W^{k}=\left(w_{i j}^{k}\right) \in \mathfrak{R}^{n \times n}, D \in \mathfrak{R}^{n \times n}$, $A \in \Re^{n \times n}(k=1,2, \ldots, r)$ are the connection weight matrices. $g(y(t))=\left[g_{1}\left(y_{1}(t), g_{2}\left(y_{2}(t), \ldots, g_{n}\left(y_{n}(t)\right] \in \mathfrak{R}^{n}\right.\right.\right.$ denotes the neuron activation function with $f(0)=0$. In this paper, we assume that activation function $g_{i}(\cdot)$ satisfies the following sector condition:

$$
\begin{aligned}
& 0 \leq \frac{g_{1}\left(\vartheta_{1}\right)-g_{2}\left(\vartheta_{2}\right)}{\vartheta_{1}-\vartheta_{2}} \leq l_{i}, \forall \vartheta_{1}, \vartheta_{2} \in \Re^{n} \\
& \vartheta_{1} \neq \vartheta_{2}, i=1,2, \ldots, n
\end{aligned}
$$

where $l_{i}(i=1,2, \ldots n)$ are positive scalars.

Time delays $\tau_{k}(t)$ and $h(t)$ are time-varying differential function and satisfy:

$$
\begin{aligned}
& 0 \leq \tau_{k}(t) \leq \tau_{k}, \dot{\tau}_{k}(t) \leq \delta_{k}<1 \\
& 0 \leq h(t) \leq h, \dot{h}(t) \leq h_{d}<1
\end{aligned}
$$

where $\tau_{d}, \delta_{k}, h$ and $h_{d}$ are constants.

The delay kernel $k_{j}$ is a real valued continuous function defined on $[0,+\infty]$ and satisfies, for each $j$, $\int_{0}^{+\infty} k_{j}(s) d s=1$. $\omega(s)$ is $m$-dimensional wiener process
(Brownian motion) on $\left(\Omega, \digamma, \digamma_{t \geq 0}\right)$. $\sigma\left(t, g(y(t)), g\left(y\left(t \quad-\quad \tau_{1}(t)\right)\right), g\left(y\left(t \quad-\quad \tau_{2}(t)\right)\right)\right.$, $\left.\ldots, g\left(y\left(t-\tau_{r}(t)\right)\right)\right) d \omega(t)$, is assumed to satisfy the following assumptions:

(A1) $\sigma(t, 0,0, \ldots, 0)=0$ and $\sigma\left(t, g(y(t)), g\left(y\left(t-\tau_{1}(t)\right)\right)\right.$, $\left.g\left(y\left(t-\tau_{2}(t)\right)\right), \quad \ldots, g\left(y\left(t-\tau_{r}(t)\right)\right)\right) d \omega(t) \quad$ is locally Lipschitz continuous and satisfies the linear growth condition.

(A2) There exist constant real matrices $\Gamma_{0}$ and $\Gamma_{i}(i=1$, $2, \ldots, r)$ such that

$$
\begin{aligned}
& \text { trace }\left[\sigma ^ { T } \left(t, g(y(t)), g\left(y\left(t-\tau_{1}(t)\right)\right), g\left(y\left(t-\tau_{2}(t)\right)\right)\right.\right. \\
& \left., \ldots, g\left(y\left(t-\tau_{r}(t)\right)\right)\right) P \sigma\left(t, g(y(t)), g\left(y\left(t-\tau_{1}(t)\right)\right)\right. \\
& \left.g\left(y\left(t-\tau_{2}(t)\right)\right), \ldots, g\left(y\left(t-\tau_{r}(t)\right)\right)\right) \\
& \leq g^{T}(y(t)) \Gamma_{0} g(y(t)) \\
& \left.+\sum_{k=1}^{r} g^{T}\left(y\left(t-t_{k}(t)\right)\right) \Gamma_{k} g\left(y-\tau_{k}(t)\right)\right) .
\end{aligned}
$$

\section{Stability Analysis}

Lemma 1. For any real vectors $a, b$ and any matrix $M>0$ with appropriate dimensions, it follows that

$$
2 a^{T} b \leq a^{T} M a+b^{T} M^{-1} b .
$$

Theorem 3.1. Assume time-varying delays $\tau_{k}(t)$ and $h(t)$ satisfy(3), the system in(1)is globally asymptotically stable, if there exist positive definite matrices $P>0$, $Q_{k}>0, T>0, Z_{i}>0, i=1,2$, positive diagonal matrix $E_{i}>0, i=1,2$ such that the following LMI holds:

$$
\Omega=\left[\begin{array}{ccc}
\Xi_{1} & \Xi_{2} & \Xi_{3} \\
\Xi_{2}^{T} & -\Xi_{4} & 0 \\
\Xi_{3}^{T} & 0 & -\Xi_{5}
\end{array}\right]<0,
$$

where

$$
\begin{aligned}
\Xi_{1} & =-P C-C P+\frac{1}{1-h_{d}}\left(Z_{1}+Z_{2}\right) \\
& +L \Gamma_{0} L+L T L+2 \sum_{k=1}^{r} L Q_{k} L \\
& +\sum_{k=1}^{r} L \frac{\Gamma_{k}}{1-\delta_{k}} L+L E_{1} L+L E_{2} L, \\
\Xi_{2} & =\left[P A P B P W^{(1)} P W^{(2)}\right. \\
& \left.\ldots P W^{(r)}\right], \\
\Xi_{3} & =\left[D P C L D P B D P W^{(1)} D P W^{(2)}\right. \\
& \left.\ldots D P W^{(r)} D P A\right], \\
\Xi_{4} & =\operatorname{diag}\left\{E_{1} T \Lambda_{1} \Lambda_{2} \ldots \Lambda_{r}\right\}, \\
\Xi_{5} & =\operatorname{diag}\left\{Z_{1} Z_{2} X_{1} X_{2} \ldots X_{r}\left(1-h_{d}\right) E_{2}\right\} .
\end{aligned}
$$

with

$\Lambda_{k}=\left(1-\delta_{k}\right) Q_{k} k=1,2, \ldots, r$,

$X_{k}=\left(1-h_{d}\right) \Lambda_{k}, k=1,2, \ldots, r$.

Proof. Consider the following lyapunov-krasoskill functional

$$
V(t)=V_{1}+V_{2}+V_{3}+V_{4},
$$


where

$$
\begin{gathered}
V_{1}=[y(t)-D y(t-h(t))]^{T} P[y(t)-D y(t-h(t))], \\
V_{2}=2 \sum_{k=1}^{r} \int_{t-\tau_{k}(t)}^{t} g^{T}(y(s)) Q_{k} g(y(s)) d s \\
\quad+\sum_{k=1}^{r} \frac{1}{1-\delta_{k}} \int_{t-\tau_{k}(t)}^{t} g^{T}(y(s)) Q_{k} g(y(s)) d s \\
V_{3}=\frac{1}{1-h_{d}} \int_{t-h(t)}^{t} y^{T}(s)\left(Z_{1}+Z_{2}+D P A E_{2}^{-1} A^{T} P D^{T}\right) \\
\times y(s) d s+\sum_{k=1}^{r} \frac{1}{1-h_{d}} \int_{t-h(t)}^{t} y^{T}(s) D P W^{(k)} \\
\times\left(1-\delta_{k}\right)^{-1} Q_{k}^{-1}\left(W^{(k)}\right)^{T} P D^{T} y(s) d s, \\
V_{4}=\sum_{j=1}^{r} e_{j}^{1} \int_{0}^{\infty} k_{j}(\delta) \int_{t-\delta}^{t} g_{j}^{2}\left(y_{j}(\delta)\right) d s d \delta \\
+\sum_{j=1}^{r} e_{j}^{2} \int_{0}^{\infty} k_{j}(\delta) \int_{t-\delta}^{t} g_{j}^{2}\left(y_{j}(\delta)\right) d s d \delta .
\end{gathered}
$$

Using It $\hat{o}^{\prime} s$ differential formula, the stochastic derivative of $\mathscr{L} V(t)$ along the trajectory of system (1) is given by

$$
\mathscr{L} V(t)=\mathscr{L} V_{1}+\mathscr{L} V_{2}+\mathscr{L} V_{3}+\mathscr{L} V_{4}
$$

where

$$
\begin{aligned}
& \mathscr{L} V_{1}=[y(t)-D y(t-h(t))]^{T} P[-C y(t) \\
& +B g(y(t))+\sum_{k=1}^{r} W^{(k)} g\left(y\left(t-\tau_{k}(t)\right)\right) \\
& \left.+A \int_{-\infty}^{t} k(t-s) g(y(s)) d s\right] \\
& +\operatorname{trace}\left[\sigma^{T} \sigma\right] \text {, } \\
& \leq-2 y^{T}(t) P C y(t)+2 y^{T}(t) P B g(y(t)) \\
& +2 y^{T}(t) P \sum_{k=1}^{r} W_{1}^{(k)} g\left(y\left(t-\tau_{k}(t)\right)\right) \\
& +2 y^{T}(t) P A \int_{-\infty}^{t} k(t-s) g(y(s)) d s \\
& +2 D y^{T}(t-h(t)) P C y(t) \\
& -2 D y^{T}(t-h(t)) P B g(y(t)) \\
& -2 D y^{T}(t-h(t)) P \sum_{k=1}^{r} W^{(k)} g\left(y\left(t-\tau_{k}(t)\right)\right) \\
& -2 D y^{T}(t-h(t)) P A \int_{-\infty}^{t} k(t-s) g(y(s)) d s \\
& +g^{T}(y(t)) \Gamma_{0} g(y(t)) \\
& \left.+\sum_{k=1}^{r} g^{T}\left(y\left(t-t_{k}(t)\right)\right) \Gamma_{k} g\left(y-\tau_{k}(t)\right)\right), \\
& \mathscr{L} V_{2} \leq 2 \sum_{k=1}^{r} g^{T}(y(t)) Q_{k} g(y(t)) \\
& -2 \sum_{k=1}^{r}\left(1-\delta_{k}\right) g^{T}\left(y\left(t-\tau_{k}(t)\right)\right) \\
& \times Q_{k} g\left(y\left(t-\tau_{k}(t)\right)\right) \\
& +\sum_{k=1}^{r} \frac{1}{1-\delta_{k}} g^{T}(y(t)) \Gamma_{k} g(y(t)) \\
& -\sum_{k=1}^{r} g^{T}\left(y\left(t-\tau_{k}(t)\right)\right) \Gamma_{k} g\left(y\left(t-\tau_{k}(t)\right)\right)
\end{aligned}
$$

$$
\begin{aligned}
& \mathscr{L} V_{3} \leq \frac{1}{1-h_{d}} y^{T}(t)\left(Z_{1}+Z_{2}+D P A E_{2}^{-1} A^{T} P D^{T}\right) \\
& \times y(t)-y^{T}(t-h(t)) \\
& \times\left(Z_{1}+Z_{2}+D P A E_{2}^{-1} A^{T} P D^{T}\right) \\
& \times y(t-h(t))+\sum_{k=1}^{r} \frac{1}{1-h_{d}} y^{T}(t) D P W^{(k)} \\
& \times\left(1-\delta_{k}\right)^{-1} Q_{k}^{-1} W^{(k) T} P D^{T} y(t) \\
& -\sum_{k=1}^{r} y^{T}(t-h(t)) D P W^{(k)}\left(1-\delta_{k}\right)^{-1} \\
& \times Q_{k}^{-1} W^{(k) T} P D^{T} y(t-h(t)), \\
& \begin{aligned}
\mathscr{L} V_{4} & \leq g^{T}(y(t)) E_{1} g(y(t)) \\
& -\left(\int_{0}^{\infty} K(t-s) g(y(s)) d s\right)^{T} \\
& \times E_{1}\left(\int_{0}^{\infty} K(t-s) g(y(s)) d s\right) \\
& +g^{T}(y(t)) E_{2} g(y(t)) \\
& -\left(\int_{0}^{\infty} K(t-s) g(y(s)) d s\right)^{T} E_{2} \\
& \times\left(\int_{0}^{\infty} K(t-s) g(y(s)) d s\right) .
\end{aligned}
\end{aligned}
$$

By applying Lemma 1, it follows that

$$
\begin{aligned}
& 2 y^{T}(t) P B g(y(t)) \\
& \leq y^{T}(t) P B T^{-1} B^{T} P y(t)+g^{T}(y(t)) T g(y(t)), \\
& 2 y^{T}(t) P W^{(k)} g\left(y\left(t-\tau_{k}(t)\right)\right) \\
& \leq y^{T}(t) P W^{(k)}\left(1-\delta_{k}\right)^{-1} Q_{k}^{-1} W^{(k) T} P y(t) \\
& +g^{T}\left(y\left(t-\tau_{k}(t)\right)\right)\left(1-\delta_{k}\right) Q_{k} g\left(y\left(t-\tau_{k}(t)\right)\right), \\
& 2 y^{T}(t) P A \int_{-\infty}^{t} k(t-s) g(y(s)) d s \\
& \leq y^{T}(t) P A E_{1}^{-1} A^{T} P y(t) \\
& +\left[\int_{-\infty}^{t} k(t-s) g(y(s)) d s\right]^{T} \\
& \quad \times E_{1}\left[\int_{-\infty}^{t} k(t-s) g(y(s)) d s\right] \\
& 2 y^{T}(t-h(t)) D P C y(t) \\
& \leq y^{T}(t-h(t)) Z_{1} y(t-h(t)) \\
& +y^{T}(t) D P C Z_{1}^{-1} C_{T} P D^{T} y(t), \\
& 2 y^{T}(t-h(t)) D P B g(y(t)) \\
& \leq g^{T}(y(t)) D P B Z_{2}^{-1} B^{T} P D^{T} g(y(t)) \\
& +y^{T}(t-h(t)) Z_{2} y(t-h(t)), \\
& 2 y^{T}(t-h(t)) D P W^{(k)} g\left(y\left(t-\tau_{k}(t)\right)\right) \\
& \leq g^{T}\left(y\left(t-\tau_{k}(t)\right)\right) \\
& \times\left(1-\delta_{k}\right) Q_{k} g\left(y\left(t-\tau_{k}(t)\right)\right)+y^{T}(t-h(t)) D \\
& \times P W^{(k)}\left(1-\delta_{k}\right)^{-1} Q_{k}^{-1} W^{(k) T} P D^{T} y(t-h(t)), \\
& 2 y^{T}(t-h(t)) D P A \int_{-\infty}^{t} k(t-s) g(y(s)) d s \\
& \leq y^{T}(t-h(t)) D P A E_{2}^{-1} A^{T} P D^{T} y(t-h(t)) \\
& {\left[\int_{-\infty}^{t} k(t-s) g(y(s)) d s\right]_{2} E_{2}} \\
& \times\left[\int_{-\infty}^{t} k(t-s) g(y(s)) d s\right], \\
& \quad{ }^{T}(t)
\end{aligned}
$$


Then, combining(9)-(14)yields, it is easy to verify that

$$
\begin{aligned}
\mathscr{L} V & \leq y^{T}(t)\left[-2 P C+\frac{1}{1-h_{d}}\left(Z_{1}+Z_{2}\right.\right. \\
& +D P A E_{2}^{-1} A^{T} P D^{T} \\
& \left.+\sum_{k=1}^{r} D P W^{(k)}\left(1-\delta_{k}\right)^{-1} Q_{k}^{-1} W^{(k) T} P D^{T}\right) \\
& +P B T^{-1} B^{T} P \\
& +\sum_{k=1}^{r} P W^{(k)}\left(1-\delta_{k}\right)^{-1} Q_{k}^{-1} W^{(k) T} P \\
& +P A E_{1}^{-1} A^{T} P+D P C Z_{1}^{-1} C^{T} P D^{T} \\
& +L \Gamma_{0} L+L D L+2 \sum_{k=1}^{r} L Q_{k} L+\sum_{k=1}^{r} L \frac{\Gamma_{k}}{1-\delta_{k}} L \\
& \left.+L E_{1} L+L E_{2} L+L D P B Z_{2}^{-1} B^{T} P D^{T} L\right] y(t) \\
& =y^{T}(t) \Omega y(t),
\end{aligned}
$$

where $\Omega$ is defined in (6). Thus, if $\Omega<0$ then the neutral system (1) is globally asymptotically stable. This completes the proof of Theorem 3.1.

Remark 1: The sufficient condition in Theorem 3.1 can ensure the global asymptotical stability of system (1), and the criterion is expressed in the form of LMI, which can be easily checked by utilizing the LMI toolbox in Matlab. Case 1: If we drop out the stochastic perturbations, and system (1) can be simplified to

$$
\begin{aligned}
& d[y(t)-D y(t-h(t))]=[-C y(t)+B(g(y(t)) \\
& +\sum_{k=1}^{r} W^{(k)} g\left(y\left(t-\tau_{k}(t)\right)\right) \\
& \left.+A \int_{-\infty}^{t} k(t-s) g(y(s)) d s\right] d t
\end{aligned}
$$

Corollary 3.2. Assume the time-varying delays $\tau_{k}(t)$ and $h(t)$ satisfy (3), system (16) is asymptotically stable if there exist positive definite matrices $P>0, Q_{k}>0$, $T>0, Z_{i}>0, i=1,2$, positive diagonal matrix $E_{i}>0, i=1,2$ such that the following LMI holds:

$$
\tilde{\Omega}=\left[\begin{array}{ccc}
\tilde{\Xi}_{1} & \Xi_{2} & \Xi_{3} \\
\Xi_{2}^{T} & -\Xi_{4} & 0 \\
\Xi_{3}^{T} & 0 & -\Xi_{5}
\end{array}\right]<0,
$$

where

$$
\begin{aligned}
& \tilde{\Xi}_{1}=-P C-C P+\frac{1}{1-h_{d}}\left(Z_{1}+Z_{2}\right)+L T L \\
& +2 \sum_{k=1}^{r} L Q_{k} L+L E_{1} L+L E_{2} L, \\
& \Xi_{2}=\left[P A P B P W^{(1)} P W^{(2)} \ldots P W^{(r)}\right], \\
& \Xi_{3}=\left[D P C L D P B D P W^{(1)} D P W^{(2)} \ldots D P W^{(r)} D P A\right], \\
& \Xi_{4}=\operatorname{diag}\left\{E_{1} T \Lambda_{1} \Lambda_{2} \ldots \Lambda_{r}\right\}, \\
& \Xi_{5}=\operatorname{diag}\left\{Z_{1} Z_{2} X_{1} X_{2} \ldots X_{r} E_{2}\right\},
\end{aligned}
$$

with

$\Lambda_{k}=\left(1-\delta_{k}\right) Q_{k}, k=1,2, \ldots, r$,

$X_{k}=\left(1-h_{d}\right) \Lambda_{k}, k=1,2, \ldots, r$.

Proof: Define a Lyapunov functional candidate for system(16)

$$
\begin{aligned}
V & =[y(t)-D y(t-h(t))]^{T} P[y(t)-D y(t-h(t))] \\
& +2 \sum_{k=1}^{r} \int_{t-\tau_{k}(t)}^{t} g^{T}(y(s)) Q_{k} g(y(s)) d s \\
& +\frac{1}{1-h_{d}} \int_{t-h(t)}^{t} y^{T}(s) \\
& \times\left(Z_{1}+Z_{2}+D P A E_{2}^{-1} A^{T} P D^{T}\right) y(s) d s \\
& +\sum_{k=1}^{r} \frac{1}{1-h_{d}} \int_{t-h(t)}^{t} y^{T}(s) D P W^{(k)} \\
& \times\left(1-\delta_{k}\right)^{-1} Q_{k}^{-1} W^{(k) T} P D^{T} y(s) d s \\
& +\sum_{j=1}^{r} e_{j}^{1} \int_{0}^{\infty} k_{j}(\delta) \int_{t-\delta}^{t} g_{j}^{2}\left(y_{j}(\delta)\right) d s d \delta \\
& +\sum_{j=1}^{r} e_{j}^{2} \int_{0}^{\infty} k_{j}(\delta) \int_{t-\delta}^{t} g_{j}^{2}\left(y_{j}(\delta)\right) d s d \delta
\end{aligned}
$$

Now, following the similar line of the proof of Theorem 3.1 , it is not difficult to show that system (16) is globally asymptotically stable in the mean square. The remaining details are omitted here. Corollary 3.2 is completed.

Case 2: If we drop out the unbounded distributed delay, and system (1) can be simplified to

$$
\begin{aligned}
& d[y(t)-D y(t-h(t))]=[-C y(t)+B(g(y(t)) \\
& +\sum_{k=1}^{r} W^{(k)} g\left(y\left(t-\tau_{k}(t)\right)\right) \\
& +\sigma\left(t, g(y(t)), g\left(y\left(t-\tau_{1}(t)\right)\right)\right. \\
& \left., g\left(y\left(t-\tau_{2}(t)\right)\right), \ldots, g\left(y\left(t-\tau_{r}(t)\right)\right)\right) d \omega(t),
\end{aligned}
$$

Corollary 3.3. Assume the time-varying delays $\tau_{k}(t)$ and $h(t)$ satisfy (3), system (19) is asymptotically stable if there exist positive definite matrices $P>0, Q_{k}>0$, $T>0$, and $Z_{i}>0, i=1,2$, such that the following LMI holds:

$$
\hat{\Omega}=\left[\begin{array}{ccc}
\hat{\Xi}_{1} & \hat{\Xi}_{2} & \hat{\Xi}_{3} \\
\hat{\Xi}_{2}^{T} & -\hat{\Xi}_{4} & 0 \\
\hat{\Xi}_{3}^{T} & 0 & -\hat{\Xi}_{5}
\end{array}\right]<0,
$$

where

$$
\begin{aligned}
& \hat{\Xi}_{1}=-P C-C P+\frac{1}{1-h_{d}}\left(Z_{1}+Z_{2}\right) \\
& +L \Gamma_{0} L+L T L+2 \sum_{k=1}^{r} L Q_{k} L \\
& +\sum_{k=1}^{r} L \frac{\Gamma_{k}}{1-\delta_{k}} L, \\
& \hat{\Xi}_{2}=\left[P B P W^{(1)} P W^{(2)} \ldots P W^{(r)}\right], \\
& \hat{\Xi}_{3}=\left[D P C L D P B D P W^{(1)} D P W^{(2)} \ldots D P W^{(r)}\right], \\
& \hat{\Xi}_{4}=\operatorname{diag}\left\{T \Lambda_{1} \Lambda_{2} \ldots \Lambda_{r}\right\}, \\
& \hat{\Xi}_{5}=\operatorname{diag}\left\{Z_{1} Z_{2} X_{1} X_{2} \ldots X_{r}\right\},
\end{aligned}
$$

with

$\Lambda_{k}=\left(1-\delta_{k}\right) Q_{k}, k=1,2, \ldots, r$,

$X_{k}=\left(1-h_{d}\right) \Lambda_{k}, k=1,2, \ldots, r$.

Proof: Define a Lyapunov functional candidate for 
system(19)

$$
\begin{aligned}
V & =[y(t)-D y(t-h(t))]^{T} P[y(t)-D y(t-h(t))] \\
& +2 \sum_{k=1}^{r} \int_{t-\tau_{k}(t)}^{t} g^{T}(y(s)) Q_{k} g(y(s)) d s \\
& +\frac{1}{1-h_{d}} \int_{t-h(t)}^{t} y^{T}(s) \\
& \times\left(Z_{1}+Z_{2}+D P A E_{2}^{-1} A^{T} P D^{T}\right) y(s) d s \\
& +\sum_{k=1}^{r} \frac{1}{1-h_{d}} \int_{t-h(t)}^{t} y^{T}(s) D P W^{(k)} \\
& \times\left(1-\delta_{k}\right)^{-1} Q_{k}^{-1} W^{(k) T} P D^{T} y(s) d s,
\end{aligned}
$$

the rest of the proof is omitted here because is straightforward from Theorem 3.1.

Case 3: If we let $D=0$, and system (1) can be simplified to

$$
\begin{aligned}
& d y(t)=[-C y(t)+B(g(y(t)) \\
& +\sum_{k=1}^{r} W^{(k)} g\left(y\left(t-\tau_{k}(t)\right)\right) \\
& \left.+A \int_{-\infty}^{t} k(t-s) g(y(s)) d s\right] d t \\
& +\sigma\left(t, g(y(t)), g\left(y\left(t-\tau_{1}(t)\right)\right),\right. \\
& \left., g\left(y\left(t-\tau_{2}(t)\right)\right), \ldots, g\left(y\left(t-\tau_{r}(t)\right)\right)\right) d \omega(t),
\end{aligned}
$$

Corollary 3.4. Assume the time-varying delays $\tau_{k}(t)$ and $h(t)$ satisfy (3), system (22) is asymptotically stable if there exist positive definite matrices $P>0, Q_{k}>0$, $T>0, Z_{i}>0, i=1,2$, positive diagonal matrix $E_{1}>0$, such that the following LMI holds:

$$
\bar{\Omega}=\left[\begin{array}{cc}
\Xi_{1} & \Xi_{2} \\
\Xi_{2}^{T} & -\Xi_{4}
\end{array}\right]<0,
$$

where

$$
\begin{aligned}
& \bar{\Xi}_{1}=-P C-C P+\frac{1}{1-h_{d}}\left(Z_{1}+Z_{2}\right)+L \Gamma_{0} L \\
& +L T L+\sum_{k=1}^{r} L \frac{\Gamma_{k}}{1-\delta_{k}} L \\
& +\sum_{k=1}^{r} L Q_{k} L+L E_{1} L, \\
& \Xi_{2}=\left[P A P B P W^{(1)} P W^{(2)} \ldots P W^{(r)}\right] \\
& \Xi_{4}=\operatorname{diag}\left\{E_{1} T \Lambda_{1} \Lambda_{2} \ldots \Lambda_{r}\right\}
\end{aligned}
$$

with

$\Lambda_{k}=\left(1-\delta_{k}\right) Q_{k}, k=1,2, \ldots, r$.

\section{A Numerical Example}

In this section, a simple example is given to demonstrate the proposed result. Consider a three-neuron stochastic neural network of neutral-type in (1) with the following parameters $(r=3)$.

$$
\begin{aligned}
& C=\left[\begin{array}{ccc}
7.2 & 0 & 0 \\
0 & 8.2 & 0 \\
0 & 0 & 8.4
\end{array}\right], \\
& B=\left[\begin{array}{ccc}
0.4 & -0.2 & 0.4 \\
0.6 & 0.2 & 0.5 \\
-0.2 & 0.8 & 0.7
\end{array}\right],
\end{aligned}
$$

$$
\begin{aligned}
& W^{1}=\left[\begin{array}{ccc}
-0.2 & 0.5 & 0.7 \\
0.3 & 0.7 & -0.2 \\
0.6 & -0.6 & 0.3
\end{array}\right], \\
& W^{2}=\left[\begin{array}{ccc}
0.1 & 0.1 & -0.2 \\
0.1 & 0.4 & 0.6 \\
0.1 & -0.1 & 0.3
\end{array}\right] \text {, } \\
& W^{3}=\left[\begin{array}{ccc}
0.2 & -0.7 & 0.1 \\
-0.7 & -0.8 & 0.7 \\
0.2 & 0.2 & -0.5
\end{array}\right] \text {, } \\
& A=\left[\begin{array}{ccc}
0.6 & 0.3 & -0.8 \\
0.2 & -0.2 & 0.1 \\
0.5 & -0.2 & 0.4
\end{array}\right] \text {, } \\
& D=\left[\begin{array}{ccc}
0.4 & 0 & 0 \\
0 & 0.4 & 0 \\
0 & 0 & 0.4
\end{array}\right] \text {, } \\
& \tau_{1}(t)=0.2+0.2 \sin (t), \\
& \tau_{2}(t)=0.2-0.2 \sin (t) \text {, } \\
& \tau_{3}(t)=0.3-0.3 \sin (t) \text {, } \\
& h(t)=0.1-0.1 \cos (t) \text {, } \\
& \Gamma_{0}=0.1 I, \Gamma_{1}=0.2 I \text {, } \\
& \Gamma_{2}=0.4 I, \Gamma_{3}=0.3 I \text {. }
\end{aligned}
$$

Take $\tau_{1}=0.4, \delta_{1}=0.2, \tau_{2}=0.4, \delta_{2}=-0.2, \tau_{3}=$ $0.6, \delta_{3}=-0.3, h=0.2, h_{d}=0.1, L=I, r=3$, applying the LMI in (6), a feasible solution is obtained as

$P=\left[\begin{array}{ccc}22.6720 & -1.6685 & 2.5446 \\ -1.6685 & 22.3430 & 1.6981 \\ 2.5446 & 1.6981 & 22.4110\end{array}\right]$

$Q_{1}=\left[\begin{array}{ccc}18.6460 & -3.8155 & 7.8362 \\ -3.8155 & 19.9655 & 5.1556 \\ 7.8362 & 5.1556 & 25.6512\end{array}\right]$,

$Q_{2}=\left[\begin{array}{ccc}17.1138 & -4.6253 & 2.2265 \\ -4.6253 & 20.8970 & -2.6437 \\ 2.2265 & -2.6437 & 15.3992\end{array}\right]$,

$\left[\begin{array}{lll}2.2265 & -2.6437 & 15.3992 \\ 7.9534 & -1.2873 & 2.2714 \\ -1.2873 & 9.8421 & 0.3159\end{array}\right]$

$Q_{3}=\left[\begin{array}{ccc}-1.2873 & 9.8421 & 0.3159 \\ 2.2714 & 0.3159 & 15.3992\end{array}\right]$,

$T=\left[\begin{array}{ccc}35.2093 & 4.6705 & -6.2582 \\ 4.6705 & 45.5331 & -7.9076 \\ -6.2582 & -7.9076 & 35.1978\end{array}\right]$,

$Z_{1}=\left[\begin{array}{ccc}62.1737 & -4.9378 & 7.0505 \\ -4.9378 & 69.2122 & 4.9682 \\ 7.0505 & 4.9682 & 71.3985\end{array}\right]$,

$Z_{2}=\left[\begin{array}{ccc}10.8685 & -2.4421 & 2.9731 \\ -2.4421 & 11.6959 & 0.0624 \\ 2.9731 & 0.0624 & 14.1976\end{array}\right]$,

$E_{1}=\left[\begin{array}{lll}20.057620 .057620 .0576\end{array}\right]$,

$E_{2}=\left[\begin{array}{lll}12.2534 & 12.2534 & 12.2534\end{array}\right]$.

Therefore, by Theorem 3.1, one can conclude that system (1) is globally asymptotically stable in the mean square.

\section{Conclusions}

In this paper, the asymptotic stability of a class of neural networks of neutral-type with multiple discrete and 
unbounded distributed delays has been studied. Based on the Lyapunov stability theory, together with the linear matrix inequality approach, a new delay-dependent sufficient condition is presented for the global asymptotic stability of the equilibrium point of the considered neural networks. In addition, the proposed stability condition is presented in terms of LMI and can be efficiently solved via standard numerical software. Finally, a numerical example has illustrated that the contributions of the main result.

\section{Acknowledgement}

This work was supported by the Doctoral Start-up Fund of East China Institute of Technology(No.DHBK2012201), the National Natural Science Foundation (No.60974090) and by the Fundamental Research Fund Funds for the Central Universities(No.CDJXS11172237).

\section{References}

[1] I. Basheer and M. Hajmeer, Journal of Microbiological Methods 43, 3-31 (2000).

[2] S. Grossberg, Neural Networks 1, 17-61, (1988).

[3] X. Liao, G. Chen and E. N, Neural Networks 15, 855-866 (2002).

[4] J. Cao and J. Wang, IEEE Transactions on Circuits and Systems I: Fundamental Theory and Applications 50, 34-44 (2003).

[5] Y. G. Liu, Z. S. You and L. P. Cao, Neurocomputing 69, 941948 (2006).

[6] W. L. Xiong and B. G. Xu, Chaos Solitons and Fractals 36, 1357-1365 (2008).

[7] X. X. Liao, Q. Luo, Z. G. Zeng and Y. X. Guo, Nonlinear Analysis-Real World Applications 9, 1535-1557 (2008).

[8] M. S. Ali and P. Balasubramaniam, Communications in Nonlinear Science and Numerical Simulation 14, 2776-2783 (2009).

[9] C. Z. Li, Y. K. Li and Y. A. Ye, Communications in Nonlinear Science and Numerical Simulation 15, 3599-3606 (2010).

[10] L. Wan and J. Sun, Phys Lett A 343, 306-31 (2005).

[11] W. H. Chen and W. X. Zheng, IEEE Transactions on Circuits and Systems I: Regular Papers 53, 644-652 (2006).

[12] Y. Ji, X. Lou and B. Cui, Chaos Solitons and Fractals 40, 344-354 (2009).

[13] M. Gao and B. T. Cui, Chaos Solitons and Fractals 40, 18231834 (2009).

[14] Z. W. Liu, H. G. Zhang and Q. L. Zhang, IEEE Trans Neural Networks 21, 1710-1718 (2010).

[15] R. S. Gau, C. H. Lien and J. G. Hsieh, International Journal of Innovative Computing Information and Control 7, 433-444 (2011).

[16] Z. Shu and J. Lam, Neurocomputing 71, 2950-2963 (2008).

[17] Y. Liu, Z. Wang and X. Liu, Neural Networks 22, 67-74 (2009).

[18] H. Huang and J. D. Cao, Nonlinear Analysis-Real World Applications 8, 646-653 (2007).
[19] M. S. Ali and P. Balasubramaniam, Communications in Nonlinear Science and Numerical Simulation 16, 2907-2916 (2011).

[20] P. Balasubramaniam and R. Rakkiyappan, Applied Mathematics and Computation 204, 680-686 (2008).

Guo-Quan Liu received his Ph.D. degree in Chonqing University in 2011 and now, he is a lecturer at the School of Mechanical and Electronic Engineering, East China Institute of Technology. His current research interests include neural networks, time-delay systems, stability theory. 